\title{
HOW ROBUST IS THE RING STAIN FOR EVAPORATING SUSPENSION DROPLETS?
}

\author{
Y. Msambwa, D. J. Fairhurst, \& F. Ouali* \\ School of Science and Technology, Nottingham Trent University, Clifton Lane, Nottingham, \\ NG11 8NS, United Kingdom \\ *Address all correspondence to F. Ouali, E-mail: fouzia.ouali@ntu.ac.uk
}

\begin{abstract}
The ring stain is commonly seen when droplets containing particles, such as coffee, are left to dry on a surface: a pinned contact line leads to outward radial flow, which is enhanced by the diverging evaporative flux at the contact line. As shown by Deegan et al. (1997) particles are swept outwards in this flow and create a ring which grows according to a simple power law with time. The final dried width and height of the ring should also be given by power laws of concentration, with both exponent equal to 0.5 provided all particles are in the ring, and the packing factor and ring profile are constant. We use suspensions of polystyrene particles in water with sizes ranging from 200 to $500 \mathrm{~nm}$ and initial concentrations $c_{0}$ from $0.009 \%$ to $1 \%$ deposited on glass substrates to investigate these scaling predictions. We vary the drying rate from 0.5 to $5 \mathrm{nl} / \mathrm{s}$ using humidity and reduced pressure, use a range of substrates to vary the initial contact angle between $5^{\circ}$ and $35^{\circ}$, and invert the droplets to change the direction of gravity. We find that for all but the very lowest pressures, the ring height follows the predicted power law, with exponent equal to $0.50 \pm 0.04$ and the ring width having an exponent of $0.33 \pm 0.05$. The discrepancy between the measured and predicted width exponent is accounted for by an observed variation of droplet radius with concentration, and the presence of particles in the center of the droplet. In addition, for low pressures (fast evaporation) the scaling laws no longer hold: the ring is much narrower and there is significant deposition in the center of the droplet, possibly due to reduced particle-enhanced pinning.
\end{abstract}

KEY WORDS: droplet, evaporation, particle, deposit, ring stain

\section{INTRODUCTION}

The work of Deegan et al. (1997) proposed a simple explanation for the common occurrence of coffee-ring stains with just two requirements: first, the triple line at the edge of the droplet must remain pinned to the substrate throughout (nearly all of) the drying process, known as constant contact area drying (Picknett and Bexon, 1977); second the evaporative flux over the droplet varies with radius $r$ away from the center of the droplet and diverges at the contact line $r=r_{d}$ following a power law:

$$
J(r) \propto\left(r_{d}-r\right)^{-\lambda},
$$

where $\lambda$ depends on the contact angle $\theta$ as $\lambda=(\pi-2 \theta) /(2 \pi-2 \theta)$. These two requirements lead to an outward flow to replenish solvent loss at the contact line, which sweeps suspended material to the contact line where it is deposited as a ring stain. By integrating the evaporation flux and balancing this with radial flow within the droplet, the authors showed that the total mass $M$ of the deposited ring should grow with power law behavior:

$$
M(t) \propto t^{2 /(1+\lambda)} .
$$

They found good experimental agreement for droplets with initial radius $r_{d}=2 \mathrm{~mm}$, contact angle $\theta=14^{\circ}$, initial concentration $c_{0} \approx 0.01 \%$, and average evaporation rate of $1.2 \mathrm{nl} / \mathrm{s}$, with roughly $90 \%$ of the particles ending up in the ring. 


\section{NOMENCLATURE}

$c_{0} \quad$ initial concentration of droplet

$D$ particle diffusion coefficient

$d_{p}$ particle diameter

$h_{r} \quad$ height of ring deposit

$J$ evaporative flux

$L \quad$ typical separation between particles

$M \quad$ mass of deposit

$m$ exponent for ring width variation

with concentration

$n \quad$ exponent for ring height variation

with concentration

$\theta \quad$ (equilibrium) contact angle

$\theta_{a}$ advancing contact angle

$\theta_{r} \quad$ receding contact angle $p$ exponent for droplet radius variation with concentration

radial coordinate

$r^{*} \quad$ rescaled radius coordinate

$r_{d}$ radius of droplet contact area

$u_{c} \quad$ critical particle speed for crystalline deposits

$V_{0} \quad$ initial droplet volume

$\dot{V} \quad$ droplet evaporation rate

$w_{r} \quad$ width of ring deposit

$z^{*} \quad$ rescaled height coordinate

$\lambda$ exponent of evaporation

$\rho$ density of a particle

$\phi \quad$ particle packing fraction in the deposit

In subsequent work by the same authors (Deegan, 1998; Deegan et al., 2000), additional relationships are found between the width $w_{r}$ and height $h_{r}$ of the ring at the moment when the liquid depins in terms of $r_{d}$ and $c_{0}$ :

$$
w_{r} \propto c_{0}^{m}, \quad h_{r} \propto w_{r}^{n / m} \propto c_{0}^{n}
$$

Experimental values of the exponents were $m=0.78 \pm 0.10$ for $0.1 \mu \mathrm{m}$ spheres and $m=0.86 \pm 0.10$ for $1 \mu \mathrm{m}$ spheres and $n / m=0.85$. The authors also point out that although they find good qualitative agreement between theory and experiment, "the theory predicts that the material arrives at the contact line earlier than it actually does," by a factor of 2. It should also be noted that although ring widths were measured directly, ring heights are only ever inferred using assumed values of the contact angle.

A simple theoretical approach, however, leads to different predictions. By conserving particles, the final mass of the deposit should be proportional to the initial volume $V$ multiplied by $c_{0}: M\left(t=t_{f}\right)=\rho V_{0} c_{0} \phi$, where $\rho$ is the density of a particle and $\phi$ is the average packing fraction in the deposit. If we assume that (i) all the particles end up in the ring, (ii) the cross-sectional shape of the deposit does not depend on $c_{0}$, and (iii) the packing fraction $\phi$ is constant, then different scaling predictions are found for both $w_{r}$ and $h_{r}$ :

$$
w_{r} \propto c_{0}^{0.5}, \quad h_{r} \propto c_{0}^{0.5} .
$$

It turns out that there are deficiencies with this simple approach. First, the packing fraction is not constant throughout the ring. For droplets containing particles with diameter $d_{p}=2 \mu \mathrm{m}$, evaporating with a constant contact area (pinned triple line), Marín et al. (2011) showed that the arrangement of particles in the ring stain is controlled by the speed with which they are carried to the periphery: in slow flow, dense, crystalline regions are built with either square or hexagonal packing; during the "rush hour" particle speeds increase above a critical speed $u_{c}$ and particles do not have time to rearrange so the ring is randomly structured. The crystalline regions are also more densely packed than the random structure, as determined by the average Voronoi area around each particle. The critical speed is found by equating the diffusive and hydrodynamic time scales and is given by

$$
u_{c} \sim \frac{L D}{d_{p}^{2}} \sim \frac{1}{c_{0}^{1 / 3}} \frac{1}{d_{p}^{3}},
$$


where $L$ is the typical separation between particles, and $D$ is the particle diffusion coefficient. There is a weak dependency on initial concentration but a much stronger dependency on particle size, which led the authors to comment that nanofluid droplets should always form crystalline deposits as the flow velocity will never exceed $u_{c}$.

Popov (2005) presents a complex calculation to predict the spatial dimensions of the ring stain, which agrees with the simple physical argument presented above, but not the measurements of Deegan. His resolution to this discrepancy is that as the depinning time is also a function of initial concentration, scaling as $\sim c_{0}^{0.26}$, more concentrated solutions will remained pinned for longer and more of the particles will end up deposited in the ring. By addition of the exponents, he recovers Deegan's $m=0.78$ exponent for ring width.

Askounis et al. (2013) investigated droplets containing particles with $d_{p}=80 \mathrm{~nm}$, evaporating with a stick-slip motion of the triple line, and observed concentric deposits due to the periodic motion of the triple line. Each circular deposit had a further structure showing four distinct regions: disordered outside, then a region with both square and hexagonal crystals, next a purely hexagonal region, and finally another disordered region. The authors proposed that as the smaller particles can approach much closer to the triple line where the evaporative flux accelerates above beyond $u_{c}$, and the effects of the disjoining pressure will be enhanced, these particles are frozen into position before they have time to crystallize. By reducing pressure, they increased particle flow and observed an increase in crystallinity. In contrast to Marín, here with much smaller particles, the flow is causing the ordering rather than disrupting it.

Yunker et al. (2011) have recently investigated the effect of varying the aspect ratio of prolate spheroidal particles on the structure and growth mechanisms of the particle deposits at the edge of a droplet. The width of the deposit was seen in all cases to increase linearly in time, but the roughness depended very sensitively on the eccentricity of the particles. Spherically-shaped particles created a compact, smooth deposit, whereas rodlike particles formed a rough, sparse, dendritic deposit. Interestingly, these suspension droplets are one of the first systems whose growth dynamics can be easily switched experimentally from one universality class to another (Yunker et al., 2011). This observation, and the other recent findings summarized above, underline the fact that there is still much to learn about the formation of the coffee ring.

In contrast, one of the aims of ongoing research into drying sessile droplets is often to remove the coffee-ring stain entirely, as many commercial processes require a uniform deposit. Several effects have been observed to achieve this goal, including: capillary forces (Weon and Je, 2010); Marangoni flow induced by surface tension gradients (Hu and Larson, 2002); electrowetting (Eral et al., 2011); and droplets smaller than a critical size (Shen et al., 2010). Many of these effects are summarized in a recent review (Sefiane et al., 2013).

Unlike the previous work mentioned above, here we seek to neither remove the coffee stain entirely, nor to thoroughly quantify its detailed structure. Instead, we investigate how robust the ring-stain scaling predictions are with respect to five experimental parameters: particle size and concentration, evaporation rate, contact angle, and droplet orientation.

\section{MATERIAL AND METHODS}

The particle suspensions used in this study were surfactant-free polystyrene spheres commercially available from Sigma-Aldrich with particle diameters of $100 \pm 30 \mathrm{~nm}, 200 \pm 30 \mathrm{~nm}$, and $500 \pm 50 \mathrm{~nm}$, supplied as $2 \%$ solids by volume and a particle density of $1.050 \mathrm{gcm}^{-3}$ at $20^{\circ} \mathrm{C}$. The suspensions were used as supplied, then diluted with deionized water to obtain samples with weight fractions equal to $0.009 \%, 0.002 \%, 0.02 \%, 0.1 \%, 0.25 \%, 0.5 \%$, and $1 \%$. The samples were left on a SRT6-Stuart roller mixer for $12 \mathrm{~h}$ to minimize the agglomeration of particles. The mixing process continued for 30 min prior to every experiment.

Three different glasses with different wetting properties were used as substrates in experiments, and the wetting properties of each were characterized by measuring equilibrium, and advancing and receding contact angles $\left(\theta, \theta_{a}\right.$, and $\theta_{r}$ ) for three different droplets using a Krüss DSA system: glass coverslips from Chance Proper, Ltd., with dimensions of $0.15 \times 24 \times 50 \mathrm{~mm}$ and $\theta=35^{\circ}, \theta_{a}=38.4 \pm 0.4^{\circ}, \theta_{r}=34.0 \pm 0.5^{\circ}$; microscope glass slides from Sail Lab Co. Ltd., (China) with $1.1 \times 25.4 \times 76.2 \mathrm{~mm}$ dimensions, and $\theta=18^{\circ}, \theta_{a}=19.1 \pm 0.3^{\circ}, \theta_{r}=16.3 \pm 0.7^{\circ}$; and microscope glass slides from Thermo Scientific (Menzel-Glaser) with $1.1 \times 26 \times 76 \mathrm{~mm}$ and $\theta=5^{\circ}$ dimension (which were not possible to measure receding angles for). Before use all substrates were cleaned by blowing with nitrogen gas to remove dust particles and any other contaminants. The zeta potentials of the glass coverslips and the

Volume 1, Number 3, 2013 
$500 \mathrm{~nm}$ particles were measured to be $-65.3 \pm 7 \mathrm{mV}$ and $-57.6 \pm 0.7 \mathrm{mV}$, respectively, using a Malvern Nano $\mathrm{S}$ Zetasizer.

Samples were placed inside a cylindrical vacuum chamber (diameter $8.6 \mathrm{~cm}$, height $5.4 \mathrm{~cm}$ ) and connected through a valve to a Cole Parmer MD 4NT vacuum pump (Baldwin et al., 2012). The pressure was recorded using a transducer (KJLC 902) interfaced with controller and readout (KPDR 900) to provide accurate control over the pressure within the chamber. Baffles were positioned within the chamber to reduce the air flow, but there is the possibility that some small air flow or vibration of the air was present due to the cyclic action of pump. On a prepared substrate, $1 \mu \mathrm{L}$ droplets of each sample concentration were carefully deposited using a Microman positive displacement pipette from Gilson, Inc. Experiments were also repeated with the droplets on the underside of the substrate. The droplets were monitored and the average drying rate calculated by recording the total drying time for each droplet and dividing the initial droplet volume by this time.

Samples were imaged after drying with an Imaging Source CCD camera with IC Capture software, using a Stocker Yale diffuse backlight (ML-045) in both reflected and transmitted modes. ImageJ was used to integrate the measured intensity around the circular deposits to create a radial profile of each deposit. Although the vertical intensity scale is only loosely related to deposit height, this measurement does enable the width of the ring $w_{r}$ to be determined with good accuracy. Optical and scanning electron microscopy were also used to examine the deposits.

The height profile of the deposits was analyzed using a Dektat 150 surface profiler with a $1 \mathrm{mg}$ scan force to reduce the chance of damage to the delicate samples. The profiler recorded a line profile of each deposit with around $3 \mu \mathrm{m}$ horizontal resolution and almost $1 \mathrm{~nm}$ vertical resolution. For each droplet, six scans were taken along diameters spaced evenly around the deposit. From the six sets of scan data, average values for the droplet radius $r_{d}$, ring height $h_{r}$, and width $w_{r}$ were determined. It should be emphasized that here we are measuring the final dried dimensions of the ring, whereas previous work (Deegan, 1998; Deegan et al., 2000) measured ring width at the moment of depinning. The entire profile was also used to calculate the fraction of particles which ended up in the ring. Representative images and profiles of deposits from two droplets with $500 \mathrm{~nm}$ particles and $c_{0}=1 \%$ drying at two different rates $(0.6$ and $3.3 \mathrm{nl} / \mathrm{s}$ ), measured using both the profiler and with imaging, are shown in Fig. 1. Very good agreement is seen for the slower drying droplet over the entire droplet. For the faster drying droplet, the position and width of the outermost ring also agree, but there are discrepancies closer to the center. The height values obtained from the profiler are more reliable and are the only data used for quantitative measurements of height.

\section{RESULTS AND DISCUSSION}

Figure 2 shows data points for the measured values for the ring width and height as a function of $c_{0}$ for droplets containing 200nm particles, at atmospheric conditions, in an upright orientation. The solid lines show power law curves with fitted exponents of $m=0.336 \pm 0.016$ for the width and $n=0.55 \pm 0.03$ for the height. Overall, similar fits were performed for 34 combinations of particle size, atmospheric pressure, contact angle, and droplet orientation. Experiments were repeated with up to four identical droplets, and the measured values of ring height and width were within the statistical uncertainties of one droplet (indicated by the error bars on Fig. 2). The fitted exponents are presented in Fig. 3. In addition, the droplet radius was observed to increase weakly with concentration, $r_{0} \propto c_{0}^{p}$, presumably due to attraction between the particles and the substrate. The values for $p$ are also plotted in Fig. 3. The average values for the three exponents are found to be $m=0.33 \pm 0.05$ for the deposit width, $n=0.50 \pm 0.04$ for the deposit height [in agreement with theoretical prediction of the height from Eq. (4)], and $p=0.03 \pm 0.01$ for the droplet radius.

As can be seen from Fig. 3, the scaling law exponents are independent of drying rate and show no additional dependency on contact angle, particle size, or droplet orientation. Colloidal particles are only weakly effected by gravity, so it is perhaps unsurprising that the orientation of the droplet does not alter the drying pattern. However, an often overlooked effect in evaporating droplets is the buoyancy of the vapor (Kelly-Zion et al., 2013), the flow of which, relative to the droplet and the substrate, will be altered by changing the relative direction of gravity. Our results, however, show that this effect is insignificant.

The constants of proportionality do show slight dependence on particle size and drying rate and a significant variation with contact angle, as flatter rings are seen as the contact angle decreases with a fixed volume. However, the 

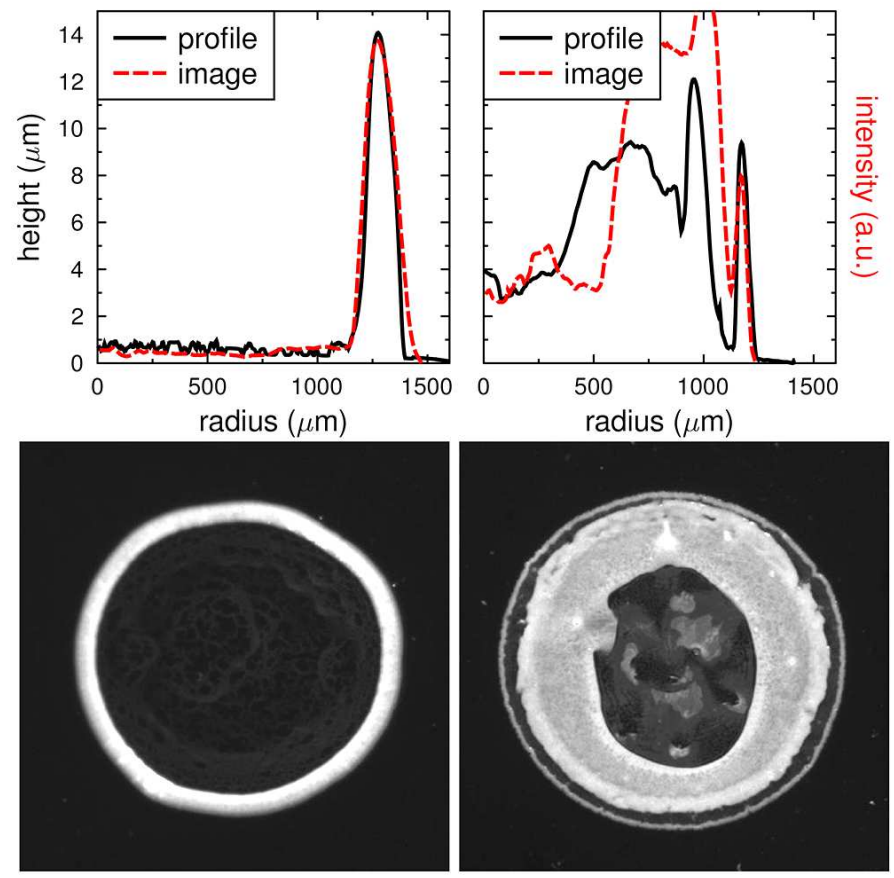

FIG. 1: Comparison of deposit profiles measured using a surface profiler (solid black lines) and imaging (red dashed lines) at two different drying rates, $\dot{V}=0.6 \mathrm{~nL} / \mathrm{s}$ (left) and $\dot{V}=3.3 \mathrm{~nL} / \mathrm{s}$ (right). The two images are shown underneath. Both droplets contained $500 \mathrm{~nm}$ particles at initial concentration $c_{0}=1 \%$.

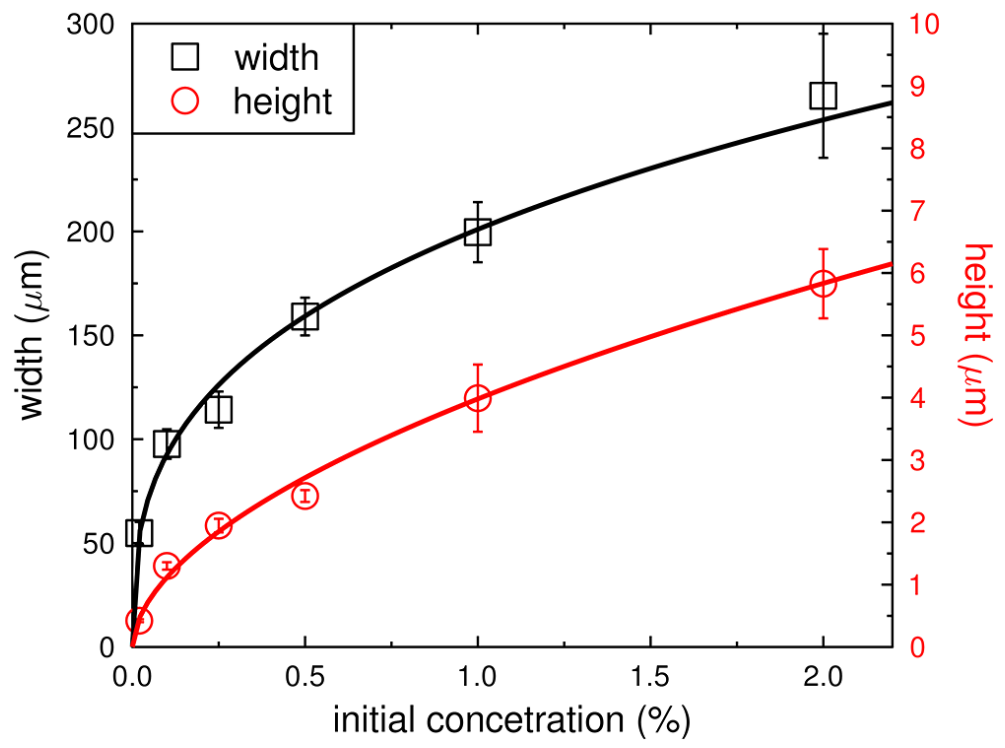

FIG. 2: Concentration dependency of the height $h_{r}$ (circles, red online) and width $w_{r}$ (black squares) of the ring stain deposited from droplets containing $200 \mathrm{~nm}$ particles, drying upright at atmospheric pressure with $\theta=5^{\circ}$. The data is fitted by the equations $w_{r}(\mu \mathrm{m})=201 c_{0}^{0.336 \pm 0.016}$ and $h_{r}(\mu \mathrm{m})=3.98 c_{0}^{0.55 \pm 0.03}$. Error bars indicate variation of the measured values within one droplet. 


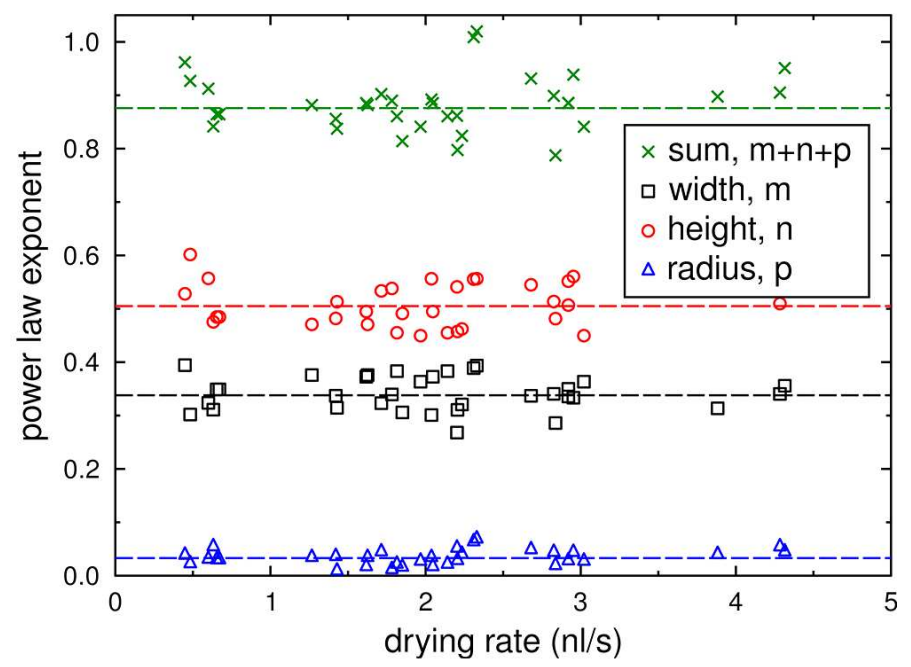

FIG. 3: Data points show the fitted values for the power law exponents describing how the ring width ( $m$, black squares), ring height $[n$, red circles (online)] and initial droplet radius [ $p$, blue triangles (online)] vary with drying rate. Also shown is the sum of all three exponents $m+n+p$. Exponents are determined for a range of particle sizes, contact angles, and orientations.

width of the ring does not vary as noticeably with contact angle. Steric repulsion at the interface, which can prevent particles from entering a region very close to the triple line, can explain the flatter deposit: an annulus containing a fixed number of particles close to the triple line will be flatter for lower contact angles. The increased droplet perimeter along which the particles are deposited may compensate for the smaller change in width.

To further investigate the validity of the scaling laws, we center the profilometer data on the peak of the deposit and rescale in the horizontal and vertical coordinates using the $1 \%$ data as the unscaled values:

$$
r^{*}=r\left(\frac{0.02}{c_{0}}\right)^{m}, \quad z^{*}=z\left(\frac{0.02}{c_{0}}\right)^{n} .
$$

As can be seen in Fig. 4, using values of $m=0.42$ and $n=0.55$, the scaled profiles are similar, although the detailed shape of the deposit shows some variation with concentration.

As discussed above, conservation of the total volume of particles dictates that the overall deposit volume should be proportional to $c_{0}$, a power law of exponent 1 . Experimentally, the deposit volume is proportional to the product of the ring height, ring width, and droplet radius. Therefore we expect that the sum of the three respective exponents $m+n+p$ should be equal to 1 . In Fig. 3 we also plot this sum, which has a value of $0.86 \pm 0.07$. Although this is close to the predicted value of unity, it is consistently smaller. The discrepancy could be accounted for by one of several explanations and is most likely to be a combination of (i) the particle packing fraction $\phi$ increases at higher concentrations so these ring deposits have misleadingly small dimension (Marín et al., 2011); (ii) the shape of the deposit varies slightly with concentration, which is not accounted for in the predictions; and (iii) a small fraction of the particles are deposited in the center of the droplet and are therefore not included in the volume calculations. Optical and electron microscopy observations show that not all of the particles are deposited in the ring. To quantify this, we used both profilometry and image analysis to estimate the fraction of particles in the ring, and for a sample with $c_{0}=1 \%$ and $\theta=35^{\circ}$, around $90 \%$ of the particles were in the ring [in agreement with previous observations (Deegan et al., 1997)] and the remaining 10\% were deposited in a monolayer of particles covering much of the initial contact area. Owing to the $3 \mu \mathrm{m}$ horizontal sensitivity of the profilometer, we were unable to reliably determine the fraction in the ring for lower concentrations.

For all samples, at the very lowest pressures investigated (10 mbar, corresponding to evaporation rates over $4 \mathrm{nl} / \mathrm{s}$ ) a much lower fraction of the particles were deposited in the peripheral ring: it has a height around $50 \%$ and width 


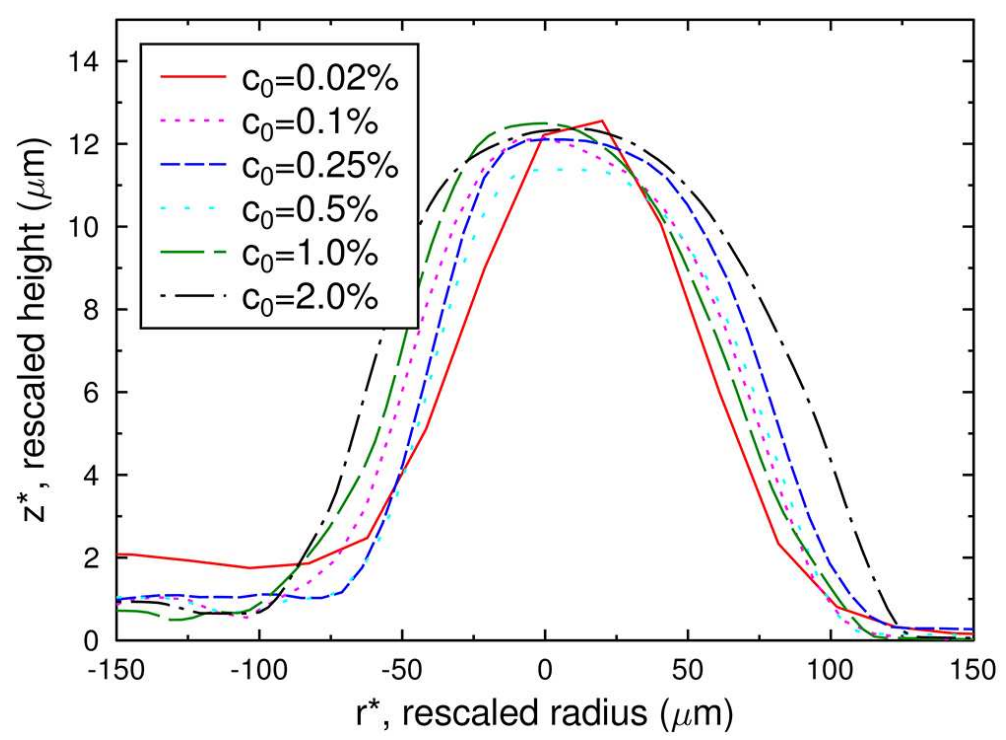

FIG. 4: Ring profile data for droplets containing $500 \mathrm{~nm}$ particles drying at $800 \mathrm{mbar}$ for a range of initial concentrations. Radius and height coordinates are rescaled with concentration using exponents $m=0.42$ and $n=0.55$, respectively. The outside of the droplet is in the positive $r^{*}$ direction.

$30 \%$, the values predicted from the scaling laws, and only around $15 \%$ of the particles are deposited here. As shown in Fig. 1, significant deposition was seen over the contact area. There appeared to be a sharp transition between ring deposition and the fast drying behavior and preliminary investigations suggest a possible mechanism: a pinned contact line leads to preferential deposition, which creates roughness at the contact line which in turn enhances the pinning. However, at the high drying rates, although the solvent is flowing rapidly to the edge as a result of the evaporation, the particles may not be swept along at the same rate, due perhaps to electrostatic interactions with other particles or with the substrate. Whatever the mechanism, fewer particles arrive at the contact line so the pinning is weaker and the interface depins earlier, reminiscent of the argument of Popov (2005). The observed sharp transition occurs due to the positive feedback in the deposition/pinning behavior. We are currently performing experimental work to investigate this further.

\section{CONCLUSIONS}

We have performed droplet evaporation experiments to determine how robust the scaling laws are which describe the height and width of the deposited ring stain. Using polystyrene particles in water on glass substrates, we have varied particle size and concentration, and droplet orientation and evaporation rate (via atmospheric pressure), and used optical and profilometric methods to determine the ring dimensions. We find that for all sizes and orientations, and all but the very fastest evaporation rates (lowest pressures), there is a robust scaling of the ring dimensions with concentration: ring width is given by $w_{r} \propto c_{0}^{0.33 \pm 0.05}$ and the height is given by $h_{r} \propto c_{0}^{0.50 \pm 0.04}$. That droplet orientation does not alter the scaling laws suggests that they should also hold under microgravity conditions. At pressures around $10 \mathrm{mbar}$, the deposit is seen across the entire contact area, which we believe is due to weaker particle-induced pinning at these high evaporation rates.

\section{ACKNOWLEDGMENT}

Y. Msambwa is funded by the Tanzanian government through the Dar Es Salaam University College of Education. 


\section{REFERENCES}

Askounis, A., Sefiane, K., Koutsos V., Shanahan, and Martin E. R., Structural transitions in a ring stain created at the contact line of evaporating nanosuspension sessile drops, Phys. Rev. E, vol. 87, p. 012301, 2013.

Baldwin, K. A., Roest, S., Fairhurst, D. J., Sefiane, K., and Shanahan, M. E. R., Monolith formation and ring-stain suppression in low-pressure evaporation of poly (ethylene oxide) droplets, J. Fluid Mech., vol. 695, pp. 321-329, 2012.

Deegan, R. D., Depostion at pinned and depinned contact lines: Pattern formation and applications, PhD Thesis, Science, 1998.

Deegan, R. D., Bakajin, O., Dupont, T. F., Huber, G., Nagel, S. R., and Witten, T. A., Capillary flow as the cause of ring stains from dried liquid drops, Nature, vol. 389, no. 6653, pp. 827-829, 1997.

Deegan, R. D., Bakajin, O., Dupont, T. F., Huber, G., Nagel, S. R., and Witten, T. A., Contact line deposits in an evaporating drop, Phys. Rev. E, vol. 62, no. 1, p. 756, 2000.

Eral, H. B., Augustine, D. M., Duits, M. H. G., and Mugele, F., Suppressing the coffee stain effect: How to control colloidal self-assembly in evaporating drops using electrowetting, Soft Matter, vol. 7, no. 10, pp. 4954-4958, 2011.

Hu, H. and Larson, R. G., Evaporation of a Sessile Droplet on a Substrate, J. Phys. Chem. B, vol. 106, no. 6, pp. 1334-1344, 2002.

Kelly-Zion, P. L., Pursell, C. J., Hasbamrer, N., Cardozo, B., Gaughan, K., and Nickels, K., Vapor distribution above an evaporating sessile drop, Int. J. Heat Mass Transfer, vol. 65, no. 0, pp. 165-172, 2013.

Marín, A. G., Gelderblom, H., Lohse, D., and Snoeijer, J. H., Order-to-disorder transition in ring-shaped colloidal stains, Phys. Rev. Lett., vol. 107, no. 8, p. 085502, 2011.

Picknett, R. G. and Bexon, R., The evaporation of sessile or pendant drops in still air, J. Colloids Interface Sci., vol. 61, pp. 336-350, 1977.

Popov, Y. O., Evaporative deposition patterns: Spatial dimensions of the deposit, Phys. Rev. E, vol. 71, no. 3, p. $036313,2005$.

Sefiane, K., Patterns from drying drops, Adv. Colloid Interface Sci., no. 0001-8686, doi: 10.1016/j.cis.2013.05.002, 2013, in press.

Shen, X., Ho, C. M., and Wong, T. S., Minimal size of coffee ring structure, J. Phys. Chem. B, vol. 114, no. 16, pp. 5269-5274, 2010.

Weon, B. M. and Je, J. H., Capillary force repels coffee-ring effect, Phys. Rev. E, vol. 82, no. 1, p. 015305, 2010.

Yunker, P. J., Still, T., Lohr, M. A., and Yodh, A. G., Suppression of the coffee-ring effect by shape-dependent capillary interactions, Nature, vol. 476, no. 7360, pp. 308-311, 2011.

Yunker, P. J., Lohr, M. A., Still, T., Borodin, A., Durian, D. J., and Yodh, A. G., Effects of particle shape on growth dynamics at edges of evaporating drops of colloidal suspensions, Phys. Rev. Lett., vol. 110, no. 3, p. 035501, 2013. 